\title{
Metagenomic Profiling and Identification of Antimicrobial Resistance Genes from Airborne Microbial Communities
}

\author{
Tamar Dickerson*1, Jonathan L. Jacobs ${ }^{1}$, Nicole Waybright ${ }^{1}$, Danielle Swales ${ }^{2}$, Peggy \\ Lowary $^{1}$, Jeanette Coffin ${ }^{1}$ and Joseph Bogan ${ }^{1}$
}

${ }^{1}$ Biosurveillance, MRIGlobal, Rockville, MD, USA; ${ }^{2}$ MRIGlobal, Charlottesville, VA, USA

\section{Objective}

To assess the temporal dynamics of airborne bacterial communities in four locations around the National Capital Region and the dispersion of antimicrobial resistant (AMR) genes present within them.

\section{Introduction}

Since the adoption of antibiotics in the early 20th century, a plethora of clinical pathogens have acquired resistance to one or more modern-day antibiotics. This has resulted in antimicrobial resistance (AMR) being recognized as a severe threat to human and animal health worldwide. Recent work has demonstrated that AMR bacteria are widely prevalent in the environment, perhaps exacerbated by the widespread use of antibiotics for clinical or agricultural purposes.

\section{Methods}

Dry air filter units were used to collect air samples daily for a period of three months at four locations inside and outside a transit center in the National Capital Region. Samples were concentrated with a Vivaspin 100,000 Dalton ultrafiltration concentrator for DNA extraction, then pooled by month and PCR amplified for 16S rRNA community profiling. Amplicon sequencing was carried out on Life Technologies (Thermo Fisher Scientific) Ion Torrent Personal Genome Machine (PGM). Additionally, pooled samples were subjected to shotgun metagenomic sequencing on the Ion Proton platform. Lastly, a functional metagenomics screen is being carried out for antibiotic resistance genes against seven antibiotics: gentamycin, chloramphenicol, ciprofloxin, trimethoprim, colistin, tetracyline and penicillin. Following confirmation of AMR, the functional gene from each positive clone will be sequenced and compared to the existing shotgun metagenomics results. This will enable the prevalence of AMR genes in each of the four airborne microbial communities to be assessed.

\section{Results}

Initial data from each of the four sites for both $16 \mathrm{~S}$ rRNA community profiling and shotgun metagenomic sequencing have shown a diverse population of environmental, human, animal and plant bacteria, with the genus Pseudomonas representing the largest proportion of sequences in both data sets. Furthermore, analysis of the shotgun metagenomics data suggests the presence of antibiotic resistance genes, whose biological functionality remains to be confirmed by further laboratory testing.

\section{Conclusions}

Preliminary results suggest that airborne microbial communities are complex and serve as a dynamic reservoir for the dispersion of antimicrobial resistance factors in the environment, potentially complicating the existing world-wide public health crisis to combat AMR pathogens.

\section{Keywords}

Biosurveillance; Airborne; Bacteria

\section{Acknowledgments}

This work is supported by a MRIGlobal Internal Research \& Development Award.

*Tamar Dickerson

E-mail: tdickerson@mriglobal.org 